\title{
Study design and rationale for biomedical shirt-based electrocardiography monitoring in relevant clinical situations: ECG-shirt study
}

\author{
Paweł Balsam ${ }^{1}$, Piotr Lodziński ${ }^{1}$, Agata Tymińska ${ }^{1}$, Krzysztof Ozierański ${ }^{1}$, \\ Łukasz Januszkiewicz ${ }^{1}$, Renata Główczyńska ${ }^{1}$, Katarzyna Wesołowska ${ }^{2}$, Michał Peller ${ }^{1}$, \\ Radosław Pietrzak ${ }^{3}$, Tomasz Książczyk ${ }^{3}$, Sonia Borodzicz ${ }^{1}$, Łukasz Kołtowski ${ }^{1}$, \\ Mariusz Borkowski ${ }^{4}$, Bożena Werner ${ }^{3}$, Grzegorz Opolski ${ }^{1}$, Marcin Grabowski ${ }^{1}$ \\ ${ }^{1} 1^{\text {st }}$ Chair and Department of Cardiology, Medical University of Warsaw, \\ Public Central Teaching Hospital in Warsaw, Poland \\ ${ }^{2}$ Department of Clinical Nursing, Medical University of Warsaw, \\ Public Central Teaching Hospital in Warsaw, Poland \\ ${ }^{3}$ Department of Pediatric Cardiology and General Pediatrics, Medical University of Warsaw, Poland \\ ${ }^{4}$ Orlik Medical Practice, Warsaw, Poland
}

\begin{abstract}
Background: Today, the main challenge for researchers is to develop new technologies which may help to improve the diagnoses of cardiovascular disease (CVD), thereby reducing healthcare costs and improving the quality of life for patients. This study aims to show the utility of biomedical shirt-based electrocardiography (ECG) monitoring of patients with CVD in different clinical situations using the Nunbo ${ }^{\circledR}$ ECG (nECG) system.

Methods: An investigator-initiated, multicenter, prospective observational study was carried out in a cardiology (adult and pediatric) and cardiac rehabilitation wards. ECG monitoring was used with the biomedical shirt in the following four independent groups of patients: 1) 30 patients after pulmonary vein isolation (PVI), 2) 30 cardiac resynchronization therapy (CRT) recipients, 3) 120 patients during cardiac rehabilitation after myocardial infarction, and 4) 40 pediatric patients with supraventricular tachycardia (SVT) before electrophysiology study. Approval for all study groups was obtained from the institutional review board. The biomedical shirt captures the electrocardiographic signal via textile electrodes integrated into a garment. The software allows the visualization and analysis of data such as ECG, heart rate, arrhythmia detecting algorithm and relative position of the body is captured by an electronic device.

Discussion: The major advantages of the $n E C G$ system are continuous ECG monitoring during daily activities, high quality of ECG recordings, as well as assurance of a proper adherence due to adequate comfort while wearing the shirt. There are only a few studies that have examined wearable systems, especially in pediatric populations.

Trial registration: This study is registered in ClinicalTrials.gov: Identifier NCT03068169. (Cardiol J 2018; 25, 1: 52-59)

Key words: remote electrocardiography, atrial fibrillation, cardiac rehabilitation, cardiac resynchronization therapy, electrophysiological study, myocardial infarction, mobile health, pulmonary veins isolation, supraventricular tachycardia, telehealth, telemedicine
\end{abstract}

Address for correspondence: Piotr Lodziński, MD, PhD, First Chair and Department of Cardiology, Medical University of Warsaw, Public Central Teaching Hospital in Warsaw, ul. Banacha 1a, 02-097 Warszawa, Poland, tel: +48 22 5992958, fax: +48 22 5991957, e-mail: piotr.lodzinski@me.com 


\section{Introduction}

Cardiovascular diseases (CVD) are associated with high healthcare costs as well as being a leading cause of mortality and hospitalization. This is an important medical, economic and social problem. Researchers today are puzzled on how to develop new monitoring technologies which improve diagnosis of CVD, reduce healthcare costs and improve the quality of life for patients. Over recent years, non-invasive wearable electronics offer new opportunities for the diagnosis and management of patients with CVD [1-3]. Biophysical signals may now be easily recorded and transmitted using wired or wireless communication and analysed anywhere, at any time.

Several reports have been published, in which wearable devices achieved very positive results with high diagnostic accuracy [3-6]. It was demonstrated that a garment-inserted electrocardiography (ECG) system can obtain similar results to those achieved with the conventional treadmill system during exercise testing, which may also include obtaining echocardiographic images [3]. Most importantly, the wearable system was invented to allow remote ECG monitoring during everyday activities. It was found useful in patients with coronary artery disease undergoing in-home cardiac rehabilitation [6]. Asimilar system (consisting of accelerometer and ECG) was also used to monitor heart rate and activities of rescuers (fire fighters and Civil Protection rescuers) during emergency interventions [4]. Kwon et al. [5] investigated a brassiere-based ECG monitoring system (CardioGuard), which showed a high degree of reliability for daily continuous ECG monitoring in women. What is important, all wearable ECG systems presented to be comfortable during tests. This ensures an adequate level of adherence, mainly due to their non-invasive nature and non-interference with normal daily life activities [3-6].

The aim of this study is to show the utility of biomedical shirt-based ECG monitoring of patients with CVD in different clinical situations using the Nuubo ECG (nECG) system.

\section{Methods}

\section{ECG platform}

The system consists of biomedical nECG shirt, an electronic device and ECG software (Fig. 1). The biomedical shirt captures the electrocardiographic signal via the textile electrodes integrated into the garment. The biomedical shirt enables noninvasive registration of a medical-quality 3-lead
ECG signal through adherence of textile electrodes to the skin. The biomedical shirt-ECG monitoring is based on BlendFix ${ }^{\circledR}$ sensor electrode technology that is capable of being used in real-time and for continuous recording. In the front of the shirt an electronic device with a memory card is attached that stores the ECG signal (as well as data form accelerometer and GPS). The battery for the device lasts for $36 \mathrm{~h}$. The device transmits information via Bluetooth to a computer for visualization and analysis of ECG, heart rate, arrhythmia detecting algorithm and relative position of the body captured by the electronic device. The Nuubo shirt is washable allowing greater comfort for the user. The platform is Conformité Européenne certified as a medical device for use in the European Union. It has been tested in patients who have undergone an exercise echocardiography test [3] and cardiac rehabilitation [6].

\section{Study design}

An investigator-initiated, multicenter, prospective observational study was carried out in a cardiology (adult and paediatric) and cardiac rehabilitation wards of two tertiary university hospitals. ECG was monitored using the biomedical shirt in the following four independent groups of patients: 1) 30 patients after pulmonary veins isolation (PVI); 2) 30 cardiac resynchronization therapy (CRT) recipients; 3) 120 patients during cardiac rehabilitation after myocardial infarction (MI), and 4) 40 pediatric patients with supraventricular tachycardia (SVT) before electrophysiology study (Fig. 2). Approval for all study groups was obtained from institutional review board. The study protocol conforms to the Standard Protocol Items: Recommendations for Interventional Trials (SPIRIT) 2013 statement (Table 1A-D) [7]. See additional file containing SPIRIT Checklist. The study received ClinicalTrials.gov number, NCT03068169.

\section{Rationale for biomedical shirt-based ECG monitoring of the blanking period and long-term effectiveness of the PVI}

Pulmonary veins isolation is a well-known method for atrial fibrillation treatment [8]. However, its efficiency is estimated at approximately $70 \%$ and depends on many factors [8]. Blanking period is referred to as an immediate 3 -month period post PVI in which early recurrences of atrial tachyarrhythmias (ERAT) are not considered clinically significant $[9,10]$. Not all ERAT lead to later recurrences, and do not necessarily represent treatment failure [10,11]. However, it is not known 


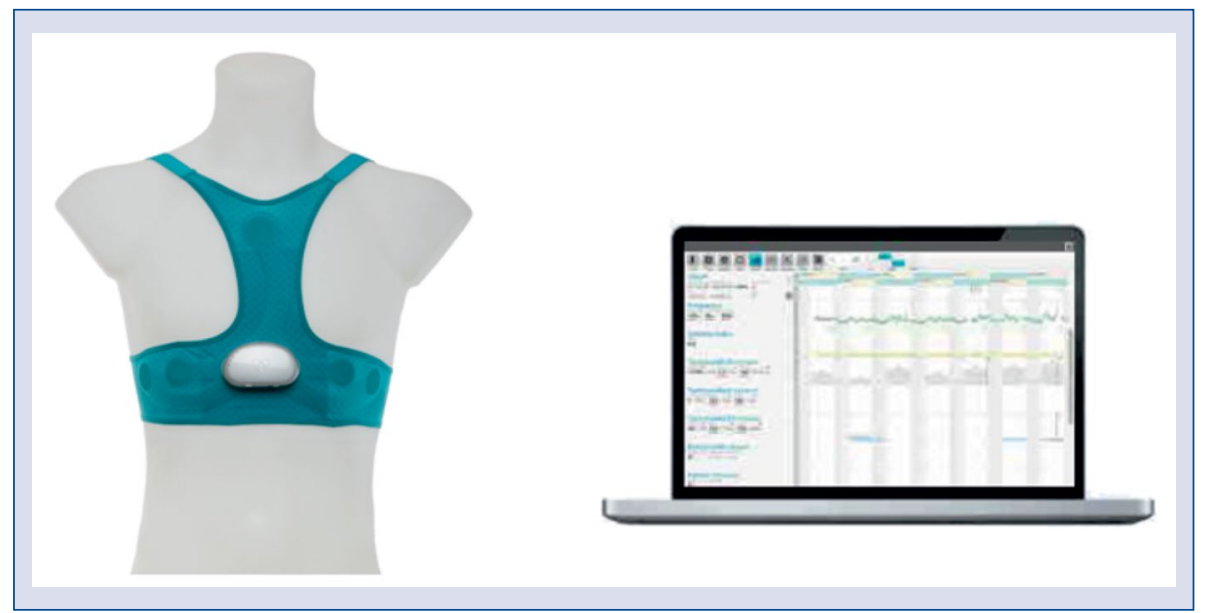

Figure 1. The wireless remote electrocardiogram platform consists of biomedical shirt, electronic device and software package.

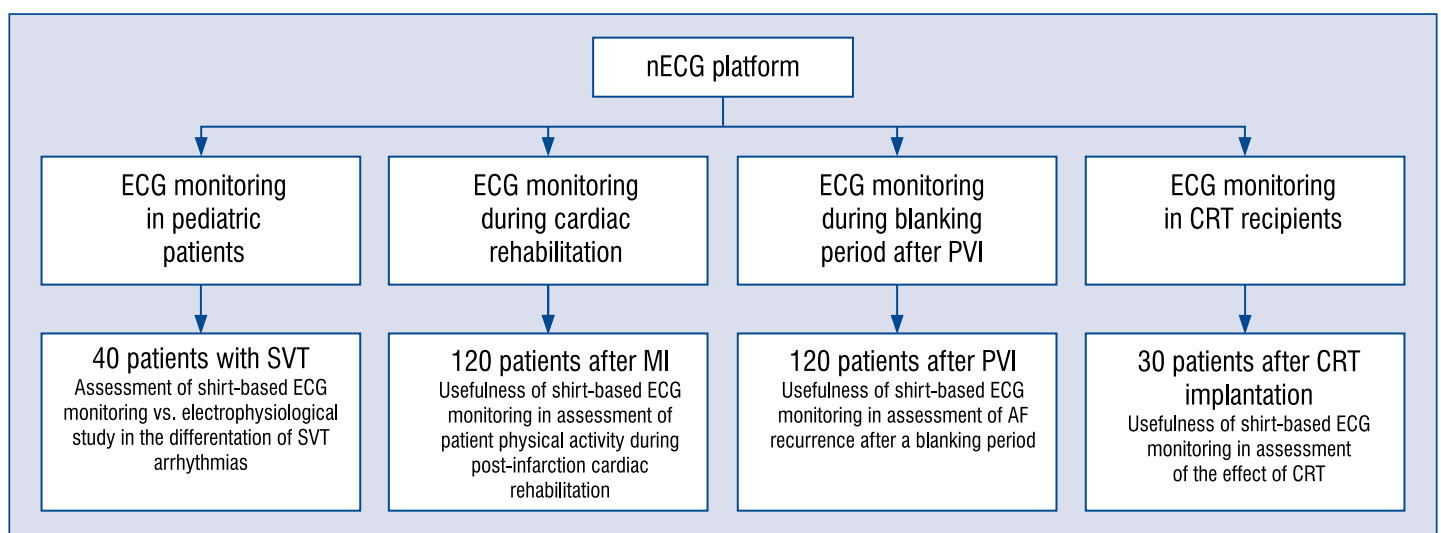

Figure 2. Study groups; AF — atrial fibrillation; CRT — cardiac resynchronization therapy; ECG - electrocardiography; $\mathrm{MI}$ - myocardial infarction; nECG - Nuubo electrocardiogram; PVI — pulmonary vein isolation; SVT — supraventricular tachycardia

whether ERAT burden in the blanking period is related to the long-term efficiency of the PVI.

\section{Rationale for biomedical shirt-based ECG monitoring of the BiV\% and physical activity in CRT recipients}

Cardiac resynchronization therapy is an established therapeutic strategy for patients with symptomatic heart failure and wide QRS complex [12]. However, approximately 1 in 3 CRT recipients do not respond to the therapy and do not derive benefit from CRT [13]. The CRT non-response problem is multifactorial. A proper qualification, impeccable procedure and appropriate programming are keys to therapy improvement [14]. Many studies have clearly shown that CRT beneficial effects can be achieved when biventricular pacing (BiV\%) is as close to $100 \%$ as possible [14]. Therefore, devices must present the correct BiV\%. Unfortunately, because of frequent improper fusion and pseudofusion beats identification, BiV\% measured by the device might be overestimated. Data on the mismatch between the BiV\% measured by the device and true $\mathrm{BiV} \%$ is scarce [15].

\section{Rationale for biomedical shirt-based ECG monitoring during cardiac rehabilitation after myocardial infarction}

Patients after acute coronary syndromes are exposed to an increased risk of recurrent MI, and therefore they should be referred for secondary prevention programs, which include cardiac rehabilitation. There is strong scientific evidence showing reduction of mortality among MI patients who 
Table 1. Recommendations for Interventional Trials (SPIRIT) 2013 statement.

A. Pediatric patients with supraventricular tachycardia (SVT).

\begin{tabular}{|c|c|c|c|c|c|}
\hline & \multicolumn{4}{|c|}{ STUDY PERIOD } & \multirow[b]{2}{*}{ Close-out } \\
\hline & Enrolment & Allocation & Monitoring & Hospitalization & \\
\hline TIME POINT & -1 week & 0 & $1^{\text {st }}$ month & $2^{\text {nd }}$ month & $3^{\text {rd }}$ month \\
\hline \multicolumn{6}{|l|}{ ENROLMENT: } \\
\hline Eligibility screen & $\mathrm{X}$ & & & & \\
\hline Informed consent & $x$ & & & & \\
\hline Allocation & & $x$ & & & \\
\hline \multicolumn{6}{|l|}{ ASSESSMENTS: } \\
\hline $\begin{array}{l}\text { Gathering medical and } \\
\text { demographic data }\end{array}$ & $x$ & & & & \\
\hline $\mathrm{nECG}$ shirt based monitoring & & & $\mathrm{X}$ & & \\
\hline Electrophysiology study (EPS) & & & & $\mathrm{X}$ & \\
\hline $\begin{array}{l}\text { Assessment of the SVT diagnosis } \\
\text { basing on the nECG shirt and EPS }\end{array}$ & & & & $x$ & \\
\hline Preparation of conclusions of the study & & & & & $x$ \\
\hline
\end{tabular}

B. Patients during cardiac rehabilitation after myocardial infarction.

\begin{tabular}{|c|c|c|c|c|c|}
\hline & \multicolumn{4}{|c|}{ STUDY PERIOD } & \multirow[b]{2}{*}{ Close-out } \\
\hline & Enrolment & Allocation & Monitoring & Assessments & \\
\hline TIME POINT & -1 week & 0 & $1^{\text {st }}$ month & After $1^{\text {st }}$ month & $3^{\text {rd }}$ month \\
\hline \multicolumn{6}{|l|}{ ENROLMENT: } \\
\hline Eligibility screen & $x$ & & & & \\
\hline Informed consent & $x$ & & & & \\
\hline Allocation & & $x$ & & & \\
\hline Randomization & $x$ & & & & \\
\hline \multicolumn{6}{|l|}{ ASSESSMENTS: } \\
\hline $\begin{array}{l}\text { Gathering medical and } \\
\text { demographic data }\end{array}$ & $x$ & & & & \\
\hline nECG shirt based monitoring & & & $\mathrm{X}$ & & \\
\hline $\begin{array}{l}\text { Performance of the standardized } \\
\text { questionnaire }\end{array}$ & & $x$ & & $x$ & \\
\hline Assessment of the study outcomes & & & & $x$ & \\
\hline Preparation of conclusions of the study & & & & & $x$ \\
\hline
\end{tabular}

C. Cardiac resynchronization therapy recipients.

\begin{tabular}{|c|c|c|c|c|c|}
\hline & \multicolumn{4}{|c|}{ STUDY PERIOD } & \multirow[b]{2}{*}{ Close-out } \\
\hline & Enrolment & Allocation & Monitoring & Assessments & \\
\hline TIME POINT & -1 week & 0 & 3 months & After $3^{\text {rd }}$ month & $4^{\text {th }}$ month \\
\hline \multicolumn{6}{|l|}{ ENROLMENT: } \\
\hline Eligibility screen & $x$ & & & & \\
\hline Informed consent & $x$ & & & & \\
\hline Allocation & & $x$ & & & \\
\hline \multicolumn{6}{|l|}{ ASSESSMENTS: } \\
\hline $\begin{array}{l}\text { Gathering medical and } \\
\text { demographic data }\end{array}$ & $x$ & & & & \\
\hline nECG shirt based monitoring & & & $x$ & & \\
\hline Assessment of the study outcomes & & & & $x$ & \\
\hline Preparation of conclusions of the study & & & & & $x$ \\
\hline
\end{tabular}


Table 1 (cont.). Recommendations for Interventional Trials (SPIRIT) 2013 statement.

D. Patients after pulmonary vein isolation.

\begin{tabular}{|c|c|c|c|c|c|c|c|}
\hline \multirow[b]{3}{*}{ TIME POINT } & \multicolumn{6}{|c|}{ STUDY PERIOD } & \multirow{3}{*}{$\begin{array}{c}\begin{array}{c}\text { Close- } \\
\text { out }\end{array} \\
\begin{array}{c}15^{\text {th }} \\
\text { month }\end{array}\end{array}$} \\
\hline & \multicolumn{2}{|c|}{ Enrolment Allocation } & \multicolumn{3}{|c|}{ Monitoring } & \multirow{2}{*}{$\begin{array}{c}\begin{array}{c}\text { Assess- } \\
\text { ments }\end{array} \\
\begin{array}{c}\text { After } 13^{\text {th }} \\
\text { months }\end{array}\end{array}$} & \\
\hline & -1 week & 0 & $\begin{array}{c}1^{\text {st }} \\
\text { month }\end{array}$ & $\begin{array}{c}7^{\text {th }} \\
\text { month }\end{array}$ & $\begin{array}{c}13^{\text {th }} \\
\text { month }\end{array}$ & & \\
\hline ENROLMENT: & & & & & & & \\
\hline Eligibility screen & $x$ & & & & & & \\
\hline Informed consent & $x$ & & & & & & \\
\hline Allocation & & $\mathrm{X}$ & & & & & \\
\hline ASSESSMENTS: & & & & & & & \\
\hline Gathering medical and demographic data & $\mathrm{X}$ & & & & & & \\
\hline Pulmonary veins isolation & & $\mathrm{X}$ & & & & & \\
\hline $\mathrm{nECG}$ shirt based monitoring & & & $\mathrm{X}$ & $\mathrm{x}$ & $\mathrm{X}$ & & \\
\hline Assessment of the study outcomes & & & & & & $x$ & \\
\hline Preparation of conclusions of the study & & & & & & & $\mathrm{X}$ \\
\hline
\end{tabular}

Table 2. Inclusion and exclusion criteria.

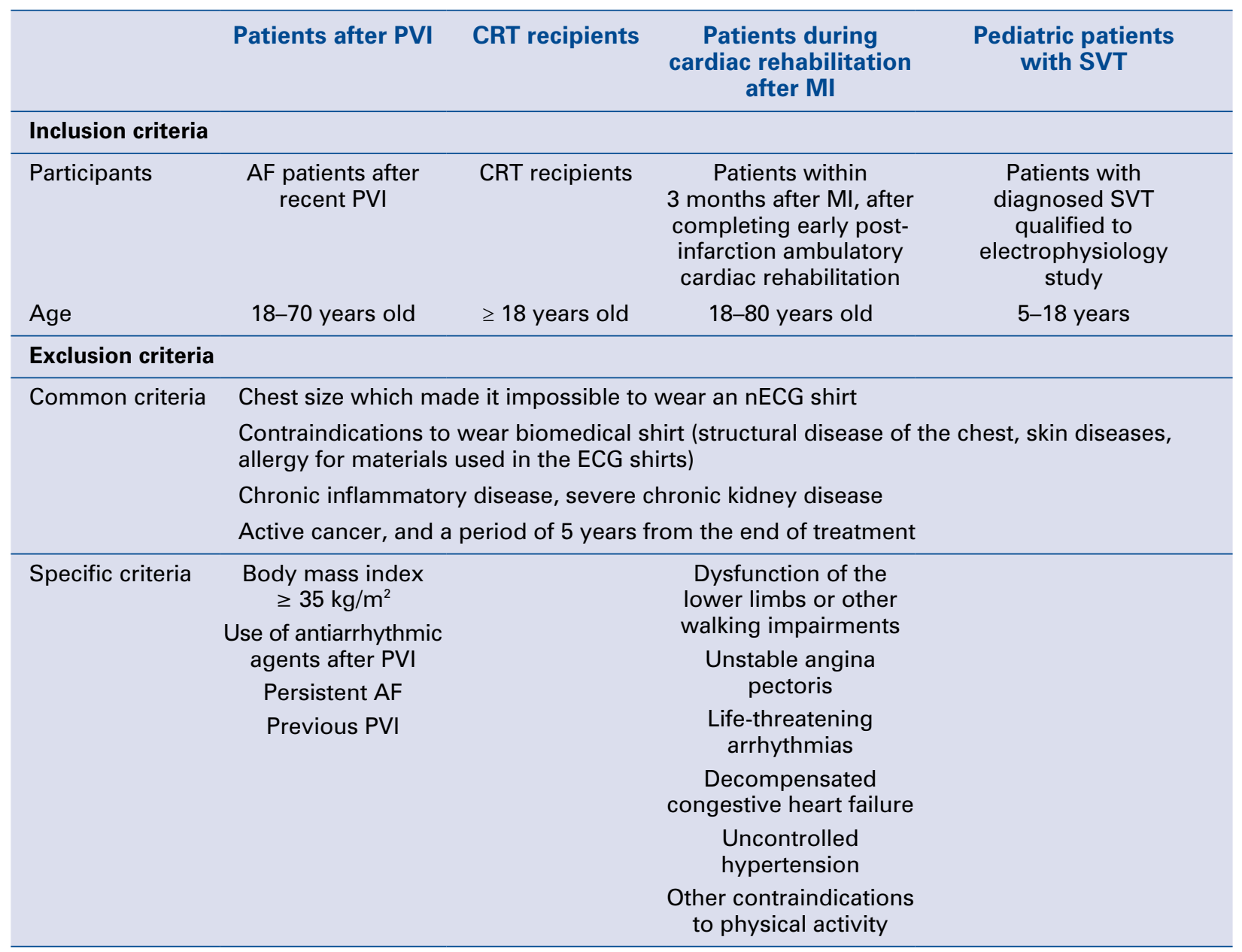

$\mathrm{AF}$ - atrial fibrillation; $\mathrm{CRT}$ - cardiac resynchronization therapy; ECG - electrocardiography; $\mathrm{MI}$ - myocardial infarction; nECG - Nuubo electrocardiogram; PVI — pulmonary vein isolation; SVT — supraventricular tachycardia 
completed a comprehensive cardiac rehabilitation track involving exercise training, diet, risk factor modification, education and psychological support $[16,17]$. Implementation of secondary prevention units in health care facilities is a key element in promoting the habits of regular physical activity among patients. Unfortunately, for many patients the access to these activities remains limited. In daily practice, a cardiac rehabilitation program is divided into three stages: 1 ) an introductory phase conducted in hospital; 2) an early rehabilitation phase run in an ambulatory or home environment; and 3) a late phase of ambulatory rehabilitation. It is assumed that the last phase of rehabilitation should last until the end of a patient's life, therefore, it is very important to promote physical activity and monitor its intensity in everyday life. This can be achieved using different motivation tools, like pedometers or $\mathrm{nECG}$ shirts in which patients are comfortable and feel safe [18-20].

\section{Rationale for biomedical shirt-based ECG monitoring to differentiate AVNRT from AVRT in pediatric patients}

Supraventricular tachycardia is one of the most common arrhythmia in the pediatric population [21]. There are two main mechanisms of SVT in pediatric patients, including atrioventricular reentry tachycardia (AVRT) and atrioventricular nodal reentry tachycardia (AVNRT). AVRT results from the presence of accessory pathways connecting the tissues of the atrium and ventricle. This enables conduction in both directions (preexcited pathways) or retrograde conduction (concealed pathways). In the AVNRT, two conducting pathways forming the reentry circuit are located within the atrioventricular node [21].

Analysis of a 12 -lead ECG is required to determine the treatment strategy and enable assessment of the SVT mechanism [22]. However, it has been demonstrated, that evaluations of ECG obtained from Holter monitors and event recorders correctly determines the mechanism of SVT only in $45 \%$ of recordings [22]. Ferdman et al. [21] published the results of the study evaluating usefulness of the AliveCor smartphone application in distinguishing between AVRT and AVNRT in pediatric populations. The authors presented that a single-lead ECG obtained with a smartphone monitor can successfully record SVT in pediatric patients, it can also predict the SVT mechanism similar topreviously published reports of Holter monitors [21]. The recently published JUNIOR SVT SCORE showed a good ability to distinguish orthodromic AVRT from AVNRT, though further validation is needed [23].

Ethics approval and consent to participate

The study protocol and written consent form were approved by the Research Ethics Board of the Medical University of Warsaw. Any modifications of the protocol that might impact patient safety, confidentiality, or other significant conduct of the study were reviewed by the Research Ethics Board before implementation.

\section{Study objectives}

Patients after PVI. The purpose of this sub-study is to examine the rationale for the use of monitoring during a blanking period and to determine whether the ERAT burden influences on long-term effectiveness of PVI. The study might demonstrate which patients with ERAT remain at risk of long-term recurrence of atrial fibrillation.

CRT recipients. The purpose of this substudy is to examine the extended monitoring in fusion and pseudo-fusion beats detection in CRT recipients to assess the true $\mathrm{BiV \%}$. The secondary aim is to assess the influence of CRT implantation on the amount of physical activity as measured by the nECG shirt.

Patients during cardiac rehabilitation after MI. The purpose of this sub-study is to assess the impact of the use of a pedometer combined with $\mathrm{nECG}$ as motivation tools for increasing the level of daily physical activity and its intensity.

Pediatric patients with SVT. The purpose of this sub-study was to create an algorithm based on $n E C G$ monitoring to differentiate between AVRT and AVNRT in pediatric patients suffering from SVT.

\section{Study populations and overall study description}

The study consisted of four independent patient groups. Patients met all inclusion criteria and no exclusion criteria, as shown in Table 2, were enrolled in the study. All participants signed informed written consent, which was obtained by a physician. In the case of children, a consent form of their legal guardians was required.

Patients after PVI. This arm of the study prospectively enrolled a cohort of 30 patients with paroxysmal AF treated with PVI. At hospital discharge, patients after PVI received the biomedical shirt. Patients wore the shirt for 1-month (taken off only for bathing) after PVI, thereafter for two 1-month periods, also after 6 and 12 months. 
CRT recipients. This cohort consisted of 30 patients scheduled for CRT implantation. Patients received the $\mathrm{nECG}$ system 1 month prior to CRT implantation. Patients wore the biomedical shirt for 1 month before implantation to measure ECG and activity and then for 1-month after CRT implantation. CRT response was assessed in the echocardiographic study performed 6 months subsequent to CRT implantation. An echocardiographic responses were defined as percent reduction in left ventricular end-diastolic volume between enrolment and 6-month follow-up. The secondary aim was to assess the influence of CRT implantation on physical activity. The device algorithms measured physical activity.

Patients during cardiac rehabilitation after MI. This arm of the study prospectively enrolled 120 patients within 3 months after MI, after completing early post-infarction ambulatory cardiac rehabilitation. The study was randomized (1:1 randomization) with a control group (60 patients) and a motivated group (60 patients).

Pediatric patients with SVT. This arm of the study prospectively enrolled a cohort consisting of 40 patients, between 5 and 18 years old diagnosed with SVT and who are qualified for electrophysiology study. Before performing electrophysiology study, patients underwent continuous ECG monitoring using a biomedical shirt. Patients wore the $\mathrm{nECG}$ shirt for 1 month or until the first SVT registration.

\section{Study endpoints}

Patients after PVI. The primary endpoint of the study was the atrial fibrillation burden during 1-month ECG monitoring after PVI. The secondary endpoint was the influence of ERAT burden in the blanking period on PVI efficacy and therefore rationale for the use of a blanking period.

CRT recipients. The primary endpoint of this sub-study was true BiV\% observed within a 3-month period. Secondary outcomes described the influence of true $\mathrm{BiV} \%$ on the CRT response and influence of CRT implantation on physical activity.

Patients during cardiac rehabilitation after MI. The primary endpoint of this arm of the study was average daily number of steps after a 1-month period of using pedometer combined with nECG. Secondary outcome was motivational value to increasing physical activity assessed by standardized questionnaire taken before and after a 1-month period of intervention.
Pediatric patients with SVT. Differentiation of AVNRT and AVRT using nECG monitoring. Results of analysis was confronted with diagnosis based on electrophysiology study.

\section{Power analysis}

Because of the observational design of the study, there was an inability to calculate the exact sample size. However, the number of patients in each arm of the study appeared to be sufficient for checking the safety and efficacy of the method.

\section{Statistical analysis}

Distribution for variables was assessed with the Shapiro-Wilk test. Normally and non-normally distributed continuous variables were presented as median and mean values, respectively. Categorical variables were presented as percentages. Statistical analysis was calculated with $\mathrm{SAS}^{\circledast}$ software, version 9.4.

\section{Discussion}

A growing number of wearable or implantable technologies with sensors and therapy delivery devices is available. Recent advances in health monitoring devices enable recording of physiological signals including ECG and physical activity during daily life. Implementation of remote monitoring devices into healthcare system provides a unique opportunity for the patients to receive medical advice without presenting to a hospital or ambulatory care units.

The major advantages of the $\mathrm{nECG}$ system include: continuous ECG monitoring during daily activities, high quality of ECG recordings, as well as assurance of a proper adherence due to adequate comfort of wearing the shirt. However, therehave been only a few studies evaluating such wearable systems. The wearable ECG monitoring adds significant value to everyday clinical practice. In this study using the nECG we will investigate several important clinical problems, as was described in the protocol.

\section{Limitations of the study}

The wearable ECG system has also some limitations derived from short battery life, dependence from the user adherence and presence of artefacts during recordings. Especially, implementing the nECG monitoring in children population may provide some challenges. Moreover, the detection of asymptomatic atrial fibrillation with 
new technologies, such as wearable shirts, has not yet been formally evaluated as an arrhythmia detection method [8].

Trial status: Patient recruitment has not yet started.

Funding: NUUBO provided without payment, the biomedical nECG shirts to carry out this study. NUUBO has no influence on the study design, data collection nor interpretation of data.

\section{Conflict of interest: None declared}

\section{References}

1. Gopalsamy C, Park S, Rajamanickam R, et al. The Wearable Motherboard?: The first generation of adaptive and responsive textile structures (ARTS) for medical applications. Virtual Reality. 1999; 4(3): 152-168, doi: 10.1007/bf01418152.

2. Despang HG, Netz S, Heinig A, et al. Wireless long-term ECG integrated into clothing. Biomed Tech (Berl). 2008; 53(6): 270-278, doi: 10.1515/BMT.2008.043, indexed in Pubmed: 19037869.

3. Perez de Isla L, Lennie V, Quezada M, et al. New generation dynamic, wireless and remote cardiac monitorization platform: a feasibility study. Int J Cardiol. 2011; 153(1): 83-85, doi: 10.1016/j.ijcard.2011.08.074, indexed in Pubmed: 21925751.

4. Curone D, Tognetti A, Secco EL, et al. Heart rate and accelerometer data fusion for activity assessment of rescuers during emergency interventions. IEEE Trans Inf Technol Biomed. 2010; 14(3): 702-710, doi: 10.1109/TITB.2010.2047727, indexed in Pubmed: 20378475.

5. Kwon S, Kim J, Kang S, et al. CardioGuard: a brassiere-based reliable ECG monitoring sensor system for supporting daily smartphone healthcare applications. Telemed J E Health. 2014; 20(12): 1093-1102, doi: 10.1089/tmj.2014.0008, indexed in Pubmed: 25405527.

6. Bravo-Escobar R, González-Represas A, Gómez-González AM, et al. Effectiveness and safety of a home-based cardiac rehabilitation programme of mixed surveillance in patients with ischemic heart disease at moderate cardiovascular risk: A randomised, controlled clinical trial. BMC Cardiovasc Disord. 2017; 17(1): 66, doi: 10.1186/s12872-017-0499-0, indexed in Pubmed: 28219338.

7. Chan AW, Tetzlaff JM, Gotzsche PC, et al. SPIRIT 2013 explanation and elaboration: guidance for protocols of clinical trials. BMJ. 2013; 346: e7586, doi: 10.1136/bmj.e7586.

8. Kirchhof P, Benussi S, Kotecha D, et al. Wytyczne ESC dotyczące leczenia migotania przedsionków w 2016 roku, opracowane we wspólpracy z EACTS. Kardiol Pol. 2016; 74(12): 1359-1469, doi: 10.5603/KP.2016.0172.

9. Lodziński P, Kiliszek M, Koźluk E, et al. Does a blanking period after pulmonary vein isolation impact long-term results? Results after 55 months of follow-up. Cardiol J. 2014; 21(4): 384-391, doi: 10.5603/CJ.a2013.0144, indexed in Pubmed: 24142681.

10. Willems S, Khairy P, Andrade JG, et al. Redefining the Blanking Period After Catheter Ablation for Paroxysmal Atrial Fibrillation: Insights From the ADVICE (Adenosine Following Pulmonary Vein Isolation to Target Dormant Conduction Elimination) Trial. Circ Arrhythm Electrophysiol. 2016; 9(8), doi: 10.1161/ /CIRCEP.115.003909, indexed in Pubmed: 27516462.
11. Andrade JG, Khairy P, Verma A, et al. Early recurrence of atrial tachyarrhythmias following radiofrequency catheter ablation of atrial fibrillation. Pacing Clin Electrophysiol. 2012; 35(1): 106-116, doi: 10.1111/j.1540-8159.2011.03256.x, indexed in Pubmed: 22054110

12. McAlister FA, Ezekowitz J, Hooton N, et al. Cardiac resynchronization therapy for patients with left ventricular systolic dysfunction: a systematic review. JAMA. 2007; 297(22): 2502-2514, doi: 10.1001/jama.297.22.2502, indexed in Pubmed: 17565085.

13. Bax J, Bleeker G, Marwick T, et al. Left ventricular dyssynchrony predicts response and prognosis after cardiac resynchronization therapy. J Am Coll Cardiol. 2004; 44(9): 1834-1840, doi: 10.1016/j.jacc.2004.08.016.

14. Prinzen FW, Vernooy K, Auricchio A. Cardiac Resynchronization Therapy: State-of-the-Art of Current Applications, Guidelines, Ongoing Trials, and Areas of Controversy. Circulation. 2013; 128(22): 2407-2418, doi: 10.1161/circulationaha.112.000112.

15. Brignole M, Auricchio A, Baron-Esquivias G, et al. 2013 ESC Guidelines on cardiac pacing and cardiac resynchronization therapy: the Task Force on cardiac pacing and resynchronization therapy of the European Society of Cardiology (ESC). Developed in collaboration with the European Heart Rhythm Association (EHRA). Eur Heart J. 2013; 34(29): 2281-2329, doi: 10.1093/ eurheartj/eht150, indexed in Pubmed: 23801822.

16. Lavie CJ, Thomas RJ, Squires RW, et al. Exercise training and cardiac rehabilitation in primary and secondary prevention of coronary heart disease. Mayo Clin Proc. 2009; 84(4): 373-383, doi: 10.1016/ /S0025-6196(11)60548-X, indexed in Pubmed: 19339657.

17. Piepoli MF, Hoes AW, Agewall S, et al. 2016 European Guidelines on cardiovascular disease prevention in clinical practice: The Sixth Joint Task Force of the European Society of Cardiology and Other Societies on Cardiovascular Disease Prevention in Clinical Practice (constituted by representatives of 10 societies and by invited experts)Developed with the special contribution of the European Association for Cardiovascular Prevention \& Rehabilitation (EACPR). Eur Heart J. 2016; 37(29): 2315-2381, doi: 10.1093/eurheartj/ehw106, indexed in Pubmed: 27222591.

18. Dylewicz P, Jegier A, Piotrowicz R, et al. Stanowisko Komisji ds. Opracowania Standardów Rehabilitacji Kardiologicznej PTK: Kompleksowa Rehabilitacja Kardiologiczna. Folia Cardiol. 2004.

19. Bravata DM, Smith-Spangler C, Sundaram V, et al. Using pedometers to increase physical activity and improve health: a systematic review. JAMA. 2007; 298(19): 2296-2304, doi: 10.1001/ /jama.298.19.2296, indexed in Pubmed: 18029834.

20. Pal S, Cheng C, Ho S. The effect of two different health messages on physical activity levels and health in sedentary overweight, middle-aged women. BMC Public Health. 2011; 11: 204, doi: 10.1186/1471-2458-11-204, indexed in Pubmed: 21453540.

21. Ferdman DJ, Liberman L, Silver ES. A Smartphone Application to Diagnose the Mechanism of Pediatric Supraventricular Tachycardia. Pediatr Cardiol. 2015; 36(7): 1452-1457, doi: 10.1007/ /s00246-015-1185-6, indexed in Pubmed: 25958154.

22. Jaeggi ET, Gilljam T, Bauersfeld U, et al. Electrocardiographic differentiation of typical atrioventricular node reentrant tachycardia from atrioventricular reciprocating tachycardia mediated by concealed accessory pathway in children. Am J Cardiol. 2003; 91(9): 1084-1089, indexed in Pubmed: 12714151.

23. Deutsch K, Stec S, Kukla P, et al. Validation of Standard and New Criteria for the Differential Diagnosis of Narrow QRS Tachycardia in Children and Adolescents. Medicine (Baltimore). 2015; 94(51): e2310, doi: 10.1097/MD.0000000000002310, indexed in Pubmed: 26705217. 